\title{
Modifiye Yapay Arı Kolonisi Algoritması ile Konsol Dayanma Duvarının Türkiye Bina Deprem Yönetmeliği 2018' e Göre Optimum Tasarımı
}

\author{
Esra Uray $^{1 *}$, Büşra Nur Çıtırık ${ }^{2}$ \\ ${ }^{1 *}$ KTO Karatay Üniversitesi, Mühendislik ve Doğa Bilimleri Fakültesi, İnşaat Mühendisliği Bölümü, Konya, Türkiye (ORCID: 0000-0002-1121-2880), \\ esra.uray@karatay.edu.tr \\ ${ }^{2}$ KTO Karatay Üniversitesi, Mühendislik ve Doğa Bilimleri Fakültesi, İnşaat Mühendisliği Bölümü, Konya, Türkiye (ORCID: 0000-0001-9294-2158), \\ citirikbusra@gmail.com
}

(3rd International Congress on Human-Computer Interaction, Optimization and Robotic Applications June 11-13, 2021)

(DOI: $10.31590 /$ ejosat.948040)

\begin{abstract}
ATIF/REFERENCE: Uray, E. \& Çıtırık, B.N. (2021). Modifiye Yapay Arı Kolonisi Algoritması ile Konsol Dayanma Duvarının Türkiye Bina Deprem Yönetmeliği 2018’e Göre Optimum Tasarımı. Avrupa Bilim ve Teknoloji Dergisi, (26), 61-67.

$\ddot{\mathrm{O} z}$

Geoteknik mühendisliğinde iki farklı zemin seviyesi arasında meydana gelen yatay kuvvetlerin stabilitesinin sağlanmasında dayanma duvarları oldukça yaygın kullanılan bir çözümdür. Bu çalışmada Türkiye Deprem Yönetmeliği 2018' e göre konsol dayanma duvarının optimum tasarımı modifiye arı kolonisi algoritması kullanılarak araştırılmıştır. Doğadan esinlenerek geliştirilen yapay arı kolonisi algoritması, arıların besin kaynağı bulma sürecini taklit eden bir sezgisel optimizasyon yöntemidir. Konsol dayanma duvarının optimum tasarımlarını elde etmek için optimizasyon analizleri yapılımış ve amaç fonksiyonu konsol dayanma duvarının maliyeti olarak alınmıştır. Duvarın geometrisinden kaynaklanan boyut limitleri, statik ve dinamik yükler etkisinde kayma, devrilme ve taşıma gücü kontrollerinin normalize edilmiş matematiksel ifadeleri algoritmaya sınırlayıcılar olarak eklenmiştir. Optimizasyon analizlerinde, temelin ön ve arka ampatman mesafeleri, gövdenin alt ve üst genişlikleri ve temel kalınlığı ayrık tasarım değişkeni olarak dikkate alınmış ve bu ayrık tasarım değiş̧ken değerleri kullanılarak tasarım kümesi oluşturulmuştur. Seçilen bölgelere göre Türkiye Deprem Haritası' ndan alınan farklı kısa periyot harita spektral ivme katsayıları ve farklı yerel zemin sınıfları için modifiye yapay arı kolonisi algoritması ile optimize edilmiş konsol dayanma duvarının minimum maliyetleri elde edilmiştir. Amaç fonksiyonunun minimum değerleri çeşitli zemin özellikleri ve deprem yüklerini içeren farklı tasarım durumları açısından karşıllaştırılmıştır. Farklı tasarım durumları dikkate alınarak elde edilen optimum sonuçlar incelendiğinde değişen özelliklerin optimum tasarıma etkisi sezgisel optimizasyon analizleri ile mantıklı olarak kısa zamanda elde edilmiştir. Modifiye yapay arı kolonisi algoritmasının konsol dayanma duvar tasarımı gibi çok bilinmeyenli karmaşık mühendislik problemlerinin çözümünde etkili ve başarılı bir yöntem olduğu görülmüştür.
\end{abstract}

Anahtar Kelimeler: Konsol Dayanma Duvarı, Deprem yükü, Modifiye arı kolonisi algoritması, Ayrık değişkenli sezgisel tasarım optimizasyonu.

\section{Optimum Design of Cantilever Retaining Wall with Modified Artificial Bee Colony Algorithm according to Turkish Building Earthquake Code 2018}

\begin{abstract}
Retaining walls are a widely employed solution in geotechnical engineering to provide the stability of the lateral forces occurring between two different soil levels. In this study, the optimum design of the cantilever retaining wall according to the Turkish Earthquake Code 2018 has been investigated via a modified bee colony algorithm. The artificial bee colony algorithm, inspired by nature, is a heuristic optimization method that mimics the process of bees tried to find food sources. The optimization analyses have been conducted to obtain economical designs and the objective function has been taken as the cost of the cantilever retaining wall. Normalized
\end{abstract}

\footnotetext{
*Sorumlu Yazar: esra.uray@karatay.edu.tr
} 
mathematical expressions of the size limits arising from the geometry of the wall and the checks of the sliding, overturning, and bearing capacity under the static and dynamic loads have been added to the algorithm as constraints. In the optimization analysis, the front and back extension length of the base, the top and bottom width of the stem, and the base thickness have been taken into consideration as the discrete design parameters and formed the design space utilizing these discrete values of parameters. The minimum costs of the cantilever retaining wall optimized by the modified artificial bee colony algorithm have been examined for different values of local soil classes and short-period map spectral acceleration coefficients taken from the Turkey Earthquake Map 2018 according to the selected regions. The minimum values of the objective function have been compared in terms of the different design cases includes variable values of soil properties and the earthquake loads. When the optimum results obtained by considering different design cases and earthquake loads have been examined, the effect of changing properties on the optimum wall design has been obtained coherently in a short time with heuristic optimization analysis. It has been observed that the modified artificial bee colony algorithm is an effective and successful method in solving complex engineering problems like the design of a cantilever retaining wall with many unknowns.

Keywords: Cantilever retaining wall, Seismic loads, Modified artificial bee colony algorithm, heuristic optimum design with discrete parameters.

\section{Giriș}

Geoteknik mühendisliğinde, farklı iki zemin kotu arasında statik ve dinamik yükleme etkileriyle meydana gelen yatay zemin yüklerin desteklenmesi probleminin çözümünde dayanma duvarları yaygın olarak kullanılmaktadır. Dayanma duvarlarının tasarımında statik ve dinamik yüklere göre elde edilen güvenli tasarımın elde edilmesinde seçilen duvar boyutlarına göre stabilite kriterlerini sağlayan tasarımın geleneksel yöntemlerle bulunması için deneme-yanılma yöntemi kullanılmaktadır. $\mathrm{Bu}$ tasarım sürecinde seçilen duvar boyutlarına göre gerekli kontroller yapılmakta ve duvar stabilitesinin sağlanmaması durumunda yeni duvar boyutlarına göre birçok deneme yapılarak güvenli dayanma duvar tasarımı elde edilinceye kadar bu süreç devam etmektedir. Zaman alan ve tekrarlayan duvar boyutlandirma sürecinden sonra elde edilen dayanma duvarı tasarımının ekonomik olduğu ve dolayısıyla yapı ağırlığına bağlı olarak yap1 üretiminde kullanılan enerji kaynaklarının oluşturduğu karbondioksit salınımı sebebiyle çevreci de olduğu garanti değildir. Ayrıca, konsol dayanma duvar tasarımının yapıldığ zemin ortamının özellikleri, desteklenecek zemin yüksekliği, zemin tabakalanma durumu ve yeraltı suyunun varlığı, yapı kullanım amacı, inşa süresi gibi faktörler tasarımı karmaşık bir mühendislik problemi haline getirmektedir. $\mathrm{Bu}$ sebeple, bahsedilen faktörlerin ve mevcut yükler etkisinin dikkate alındığı durumda stabilite kriterleri sağlanmış güvenli, minimum yapı ağırlığına sahip ekonomik ve çevreci tasarımın mümkün olan en kısa zamanda elde edilmesinde optimizasyon yöntemlerinin kullanılması ön plana çıkmaktadır.

Birçok araştırmacının günümüz çok bilinmeyenli karmaşık mühendislik problemlerinin çözümünde sezgisel optimizasyon yöntemlerinin yaygın olarak kullanılması artan popülerliğini açıklamaktadır. Sezgisel optimizasyon yöntemlerinin gerçek çözümü garanti etmemekle birlikte kısa zamanda optimum sonuca yakınsayan güçlü ve etkili bir yöntem olması, basit yapısı ve kolaylıkla uygulanabilmesi sebebiyle konsol dayanma duvarlarının optimum tasarımlarının elde edilmesi ve maliyet analizlerinin yapılmasında yaygın olarak kullanılmaktadır. Benzetimli tavlama algoritması, parçacık sürü optimizasyonu, büyük patlama-büyük büzülme algoritması, ateş böceği algoritması, öğretme-öğrenme temelli optimizasyon algoritması, harmoni arama algoritması, gri kurt algoritması, yapay arı kolonisi algoritması ile yapılan bilimsel araştırmalar literatürde bulunan örneklerdir (Camp \& Akin, 2012; Ceranic et al., 2001; Chau \& Albermani, 2003; Dağdeviren \& Kaymak, 2018; Kalemci et al., 2020; Khajehzadeh et al., 2010; Sheikholeslami et al., 2014; Temur \& Bekdas, 2016; Uray et al., 2021). Konsol dayanma duvar tasarımında dinamik yük etkisi deprem bölgelerinde yapılacak olan tasarımlar için göz önünde bulundurulması gereken bir durumdur. Dayanma duvarlarının tasarımında deprem etkisi için Türk deprem yönetmeliklerinde belirtilen tasarım kriterlerinin dikkate alındığı birçok çalışma yapılmış (Arslan et al., 2018; Bilgin, 2006; Dalyan et al., 2020; Y1ldırım, 2004) ve bu çalışmalarda deprem etkisi ile dayanma duvarı-zemin arasında meydana gelen etkileşim etkisinde duvar stabilitesinin kontrol edilmesinde etkili olan yatay zemin kuvvetinin belirlenmesi ile ilgili detaylar stabilite tahkikleri ile birlikte verilmiştir. Dayanma duvar tasarımında dikkate alınan deprem etkisi ile birlikte farklı zemin koşullarında güvenli ve ekonomik tasarımların elde edilmesi de karmaşık bir mühendislik problemi olup optimum tasarımların sezgisel yöntemler kullanılarak elde edilmesi mümkündür (Aydogdu, 2017; Kaveh et al., 2021; Kayhan \& Demir, 2018; Ravichandran et al., 2021).

$\mathrm{Bu}$ çalışmada dayanma yapısı olarak yaygın uygulamaları olan konsol dayanma duvarının (KDD) optimum tasarımı modifiye arı kolonisi algoritması (Akay \& Karaboga, 2012; Karaboga \& Akay, 2011) kullanılarak araştırılmıştır. Türkiye Bina Deprem Yönetmeliği (TDBY18) 'nde belirtilen stabilite kriterleri, Türkiye Deprem Tehlike Haritaları (TDTH, 2018)' ndan alınan farklı deprem etkileri ve zemin özelliklerini kapsayan farklı tasarım durumlarına göre optimum KDD tasarımları elde edilmiştir.

\section{Materyal ve Metot}

\subsection{TDBY18' e göre Konsol Dayanma Duvarı Optimizasyon Tasarım Probleminin Formülasyonu}

TBDY (2018)' e göre konsol dayanma duvarının (KDD) optimum tasarımının araştırılmasında duvara etkiyen yükler statik durum için aktif zemin basıncı $\left(\mathrm{P}_{\mathrm{as}}\right)$, statik ve dinamik durumlar için toplam zemin basıncı $\left(\mathrm{P}_{\mathrm{t}}\right)$ ve dinamik durum için aktif zemin basıncı $\left(\mathrm{P}_{\mathrm{ae}}\right)$ stabilite tahkiklerinde dikkate alınmıştır. Bu yüklere göre gerçekleştirilen stabilite kontrolleri için TBDY18 (2018) dikkate alınmış ve temel zeminin nihai taşıma kapasitesinin $\left(\mathrm{q}_{\mathrm{u}}\right)$ hesaplanmasında Meyerhof (1963) tarafindan önerilen genel taşıma kapasitesi ifadesi kullanılmıştır (Das, 2007). Optimum KDD tasarımının stabilite tahkiklerinde dikkate alınan ayrık tasarım değişkenleri (duvar boyutları) belli bir artım miktarı ile alt ve üst limit değerleri Tablo 1' de ve duvara etki eden yüklerle birlikte şematik KDD Şekil 1' de verilmiştir. Duvar boyutlarının uygulanabilir tamsayı değerlerine sahip olması için tasarım değişkenlerine karşılık gelen duvar boyutları ayrık değişken olarak alınmıştır. 
Tablo 1. Ayrık tasarım değişkenleri

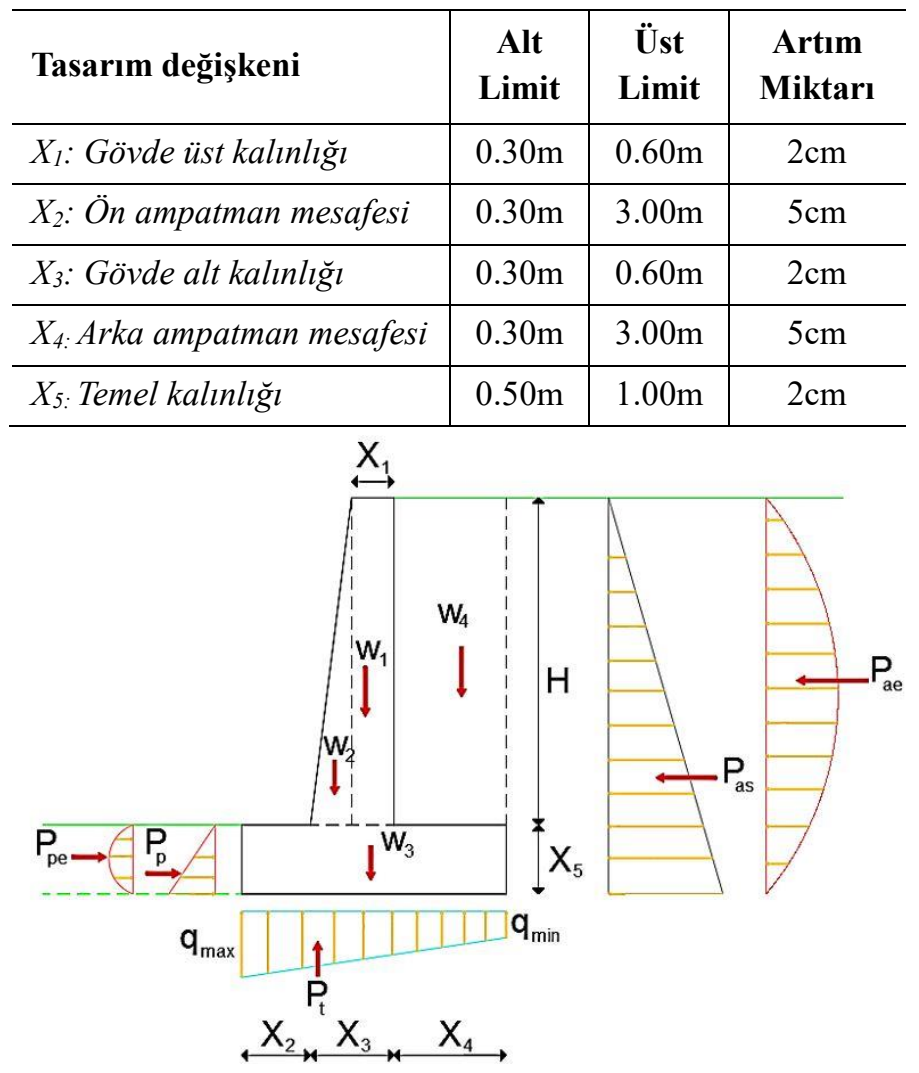

Şekil 1. KDD tasarım değişkenleri ve etki eden yükler

KDD tasarımı depremli ve depremsiz durum için kayma, devrilme ve taşıma gücü stabilite tahkiklerini sağlaması gerekmektedir. Stabilite kriterlerini sağlayan optimum KDD tasarımlarının araştırılmasında kullanılan depremsiz ve depremli durum için kayma, devrilme ve taşıma gücü tahkiklerini kapsayan stabilite sınırlayıcıları ile duvarın geometrisinden kaynaklanan geometrik sınırlayıcıların normalize edilmiş matematiksel ifadeleri ve amaç fonksiyonu Tablo 2' de verilmiştir. Amaç fonksiyonunda verilen $c_{b}$ katsayıs1 $\mathrm{C} 30 / 35$ beton birim maliyet değeri olup güncel fiyatlara göre yaklaşık olarak $290 \mathrm{TL} / \mathrm{m}^{3}$ ve beton birim hacim ağırlığ $1, \gamma_{\mathrm{b}}=24 \mathrm{kN} / \mathrm{m}^{3}$ olarak alınmıştır.

Stabilite tahkiklerinde dolgu zemini için kohezyonsuz zemin olarak dikkate alınırken ve temel zemini için drenajsız kayma mukavemeti, $c_{\mathrm{u}}=250 \mathrm{kPa}$, birim hacim ağırlık, $\gamma_{\mathrm{t}}=19 \mathrm{kNm}^{3}$, $c^{\prime}=70 \mathrm{kPa}$ ve $\phi^{\prime}=25^{\circ}$ olan kohezyonlu kil zemin kabul edilmiştir.

Tablo 2.Optimizasyon probleminin tanımlanmasında dikkate alınan sınırlayıcılar ve amaç fonksiyonu

\section{Sınırlayıcılar Normalize edilmiş matematiksel ifadeler}

Kayma tahkiki $g_{x}(1)=1-\left(\Sigma V \tan \left(k_{1} \phi_{t}\right)+\left(X_{2}+X_{3}+X_{4}\right) k_{2} c_{t}+P_{p}\right) / 1.5 P_{a s}, \Sigma V=W_{1}+W_{2}+W_{3}+W_{4}$

Devrilme tahkiki

$$
g_{x}(2)=1-M_{r} / 1.5 M_{o}, M_{r}=\Sigma W_{i} x w_{i}, M_{o}=P_{a s}\left(H+X_{5}\right) / 3, P_{a s}=\gamma_{d}\left(H+X_{5}\right) K_{a}
$$

Taşıma gücü

$g_{x}(3)=1-\frac{q_{u}}{3 q_{\max }}, g_{x}(4)=\frac{6 e}{X_{2}+X_{3}+X_{4}}, q_{\min }=\frac{\Sigma V}{X_{2}+X_{3}+X_{4}}-\left(1 \pm \frac{6 e}{X_{2}+X_{3}+X_{4}}\right), e=\frac{X_{2}+X_{3}+X_{4}}{2}-\frac{M_{r}-M_{o}}{\Sigma V}$ Kayma tahkiki $g_{x}(5)=\frac{P_{a s}+P_{a e}+\Sigma V k_{h}}{\left(X_{2}+X_{3}+X_{4}\right) c_{u t} / 1.1+0.3\left(k_{p}\left(1-k_{v}\right) P_{p}\right) / 1.4}-1, k_{h}=0.4 \times \frac{S_{D S}}{r}, k_{v}=0.5 \times k_{h}$

Devrilme tahkiki $g_{x}(6)=1-\frac{\Sigma W_{i} x w_{i}\left(1-k_{v}\right)}{1.3\left[\left(H+X_{5}\right)\left(0.5 P_{a e}+0.33 P_{a s}\right)+k_{h} \Sigma W_{i} y_{i}\right]}, P_{t}=K a(1 \pm k v)\left[\frac{1}{2} \gamma x H^{2}\right], P_{a e}=P_{t}-P_{a s}$

Taşıma gücü $\quad g_{x}(7)=\Sigma V /\left[\left(X_{2}+X_{3}+X_{4}\right) q_{u}\right]-1$

Geometrik (Statik

ve dinamik durum)

$g_{x}(8)=\left(X_{2}+X_{3}\right) /\left(X_{2}+X_{3}+X_{4}\right)-1, g_{x}(9)=X_{1} / X_{3}-1$

Amaçfonksiyonu $\quad f_{\min }=c_{b}\left(W_{1}+W_{2}+W_{3}\right) / \gamma_{b}$

\subsection{Modifiye Yapay Arı Kolonisi Algoritması (MYAKA)}

Karaboğa (2005) tarafindan önerilen yapay arı kolonisi algoritmas1 (YAKA) hayatta kalmak ve beslenme, savunma ve göç gibi temel yaşam gereksinimlerini karşılamak için 
popülasyondaki bireyler arasındaki iletişimi kapsayan sürü zekasını temel alan sezgisel optimizasyon algoritmalarından biridir. Şekil 2' de arıların besin kaynağı arama çevrimi ve algoritma akış diyagramı verilmiştir(Karaboğa, 2014).

Algoritma parametreleri olan işçi arı sayısı (NEB), gözcü arı sayısı (NOB), besin kaynağı sayısı (NFS), maksimum iterasyon sayısı (maxiter) ve limit ile başlangıç besin kaynakları $\left(\mathrm{x}_{\mathrm{ij}}\right)$ belirlenir (Akay \& Karaboga, 2012). Besin kaynağına gönderilen işçi arılar mevcut besin kaynağının komşuluğunda yeni besin kaynağı $\left(\mathrm{v}_{\mathrm{ij}}\right)$ belirleyerek besin kaynağının uygunluk değerini belirler (Eşitlik 1).

$$
\begin{aligned}
& x_{i j}=x_{j}^{\min }+\operatorname{rand}(0,1)\left(x_{j}^{\max }-x_{j}^{\min }\right)(i=1, \ldots, N F S, j=1, \ldots, N) \\
& v_{i j}=\left\{\begin{array}{c}
x_{i j}+\phi_{i j}\left(x_{i j}-x_{k j}\right), \operatorname{rand}(0,1)<M R \\
x_{i j}, \operatorname{rand}(0,1) \geq M R
\end{array}\left(\phi_{i j}=[-1,1]\right)\right.
\end{aligned}
$$

Burada $\mathrm{N}$, toplam tasarım değişkeni sayışıma ve rand $(0,1) 0$ ile 1 arasında rastgele seçilen sayıya karşılık gelmektedir. Tasarım değişkenlerinin alt ve üst sınırları $\mathrm{x}_{\mathrm{j}}^{\min }{ }^{\text {me }} \mathrm{x}_{\mathrm{j}}{ }^{\max }$ ile tanımlanmıştır. $\mathrm{x}_{\mathrm{ij}}, \mathrm{i}^{\prime}$ inci besin kaynağı için seçilen $\mathrm{j}$ ' inci tasarım değişkenine, $\mathrm{k}$, 1 ile NFS arasında rastgele seçilen bir değere karşılık gelmektedir.
Modifikasyon oranı, MR, yeni bir kaynağın geliştirilip geliştirilmediğini kontrol eden bir parametredir. MR değerinin 0.30-0.80 arasında alınması önerilmiştir (Akay ve Karaboğa, 2012). Yeni besin kaynağının uygunluk değeri (fitness $s_{i}$ ) amaç fonksiyon değeri $\left(f_{i}\right)$ kullanılarak belirlenir. Belirlenen uygunluk değeri ve sınırlayıcılarının ceza değerleri dikkate alınarak $x_{i}$ ve $v_{i}$ arasından daha iyi olan besin kaynağı seçimi yapılmaktadır. Sınırlayıcıların ceza değerinin (violation ${ }_{i}$ ) belirlenmesinde Deb's(2000) kuralları uygulanmıştır. Daha iyi uygunluk değerine sahip olan besin kaynağı eski kaynak yerine hafizaya alınıp ve yeni çözüm geliştirildiği için çözüm geliştirememe sayacı sıfırlanmaktadır. Yeni besin kaynağının daha iyi uygunluk değerine sahip olmaması durumunda çözüm geliştirememe sayacı bir artırılmaktadır. Görevli işçi arılar kovan çevresinde meydana getirdikleri besin kaynağı arayışından sonra buldukları besin kaynakları ile ilgili bilgileri gözcü arılarla paylaştıktan sonra gözcü arılar besin kaynaklarının nektar miktarı, konumu, kalitesi gibi bilgileri değerlendirerek gidecekleri besin kaynağını seçerler. Gözcü arılar seçme işleminde besin kaynağı için belirlenen uygunluk değerine göre hesaplanmış olasılık değerini $\left(\mathrm{p}_{\mathrm{i}}\right)$ dikkate alırlar (Eşitlik 2).

$$
\text { fitness }_{i}=\left\{\begin{array}{c}
1 /\left(1+f_{i}\right), f_{i} \geq 0 \\
1+\left|f_{i}\right|<0
\end{array}, p_{i}=\left\{\begin{array}{c}
0.5+0.5\left(\text { fitness }_{i} / \sum_{j=1}^{N F S} \text { fitness }_{j}\right), \text { feasible çözüm } \\
0.5\left(1-\text { violation }_{i} / \sum_{j=1}^{N F S} \text { violation }_{j}\right), \text { feasible olmayan çözüm }
\end{array}\right.\right.
$$
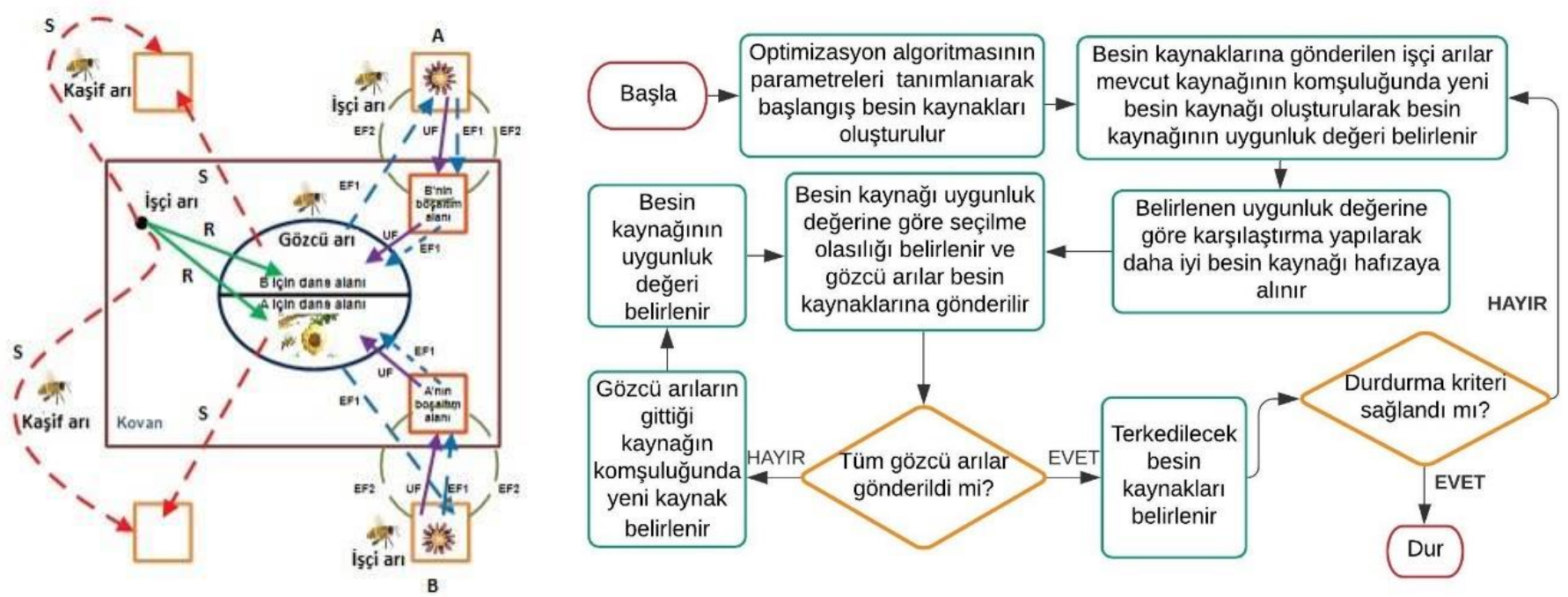

Şekil 2. Arıların besin kaynă̆ı arama süreçleri ve algoritma akış diyagramı

$[0,1]$ aralığında üretilen rastgele sayı $\mathrm{p}_{i}^{\prime}$ den büyükse, gözcü arılar işçi arılar gibi yeni bir besin kaynağ $1\left(\mathrm{v}_{i}\right)$ üretirler. Yeni besin kaynağı ile eski besin kaynağı karşılaştırılarak daha iyi ya da daha kötü besin kaynağı olma durumuna göre çözüm geliştirmeme sayacı güncellenir. Bu işlem tüm gözcü arıları besin kaynaklarına gönderilinceye kadar devam eder. İşçi ve gözcü arılar döngüleri tamamladığında bir besin kaynağındaki nektarın bitip bitmediği çözüm geliştirmeme sayacı kontrol edilerek belirlenir. Çözüm geliştireme sayacı limit değerinin üstünde olması durumunda nektarı biten kaynak terkedilir ve görevli arı kâşif arıya dönüşerek rastgele besin kaynağı arama süreci başlar. $\mathrm{Bu}$ döngü, mevcut yineleme numarası maksimum yineleme sayısına ulaşıncaya kadar devam eder ve ardından algoritma sona erer.

\section{Araştırma Sonuçları ve Tartışma}

Şekil 1' de verilen tasarım değişkenleri ve tasarımda stabilite kriterlerinin araştırılmasında dikkate alınacak olan etki eden yüklerin verildiği KDD tasarımının optimum maliyeti MYAKA ile araştırılmıştır. Optimum maliyetlerin araştırılmasında farklı yerel zemin sinıfi (ZA, ZB, ZC, ZD, ZE) ve harita spektral ivme katsayısı $\left(\mathrm{S}_{\mathrm{S}}\right)$ değerlerini kapsayan on farklı tasarım durumu dikkate alınmıştır. Optimizasyon analizlerinde duvar arka dolgusunda kullanılmak üzere yerel zemin sınıfları için kabul edilen zemin özellikleri olan dolgu zemin kayma direnci açısı $\left(\emptyset_{\mathrm{ds}}\right)$ ve birim hacim ağırlık $\left(\gamma_{\mathrm{ds}}\right)$ olacak şekilde Tablo 3' te verilmiştir. TBDY (2018)' e göre depremli durumda KDD tasarımında izin verilen yer değiştirme miktarları (r) dayanma yapısı tipine göre tanımlanmış olup bu çalışmada $r$ değeri 1.5 
olarak dikkate alınmıştır. TDTH (2018)' de verilen farklı bölgelerin depremselliği ve bu bölgelerin farklı yerel zemin sınıfı değerlerive deprem yer hareketi düzeyi DD-2 için elde edilen deprem raporları incelenerek şiddetli ve hafif şiddetli deprem durumlarına göre $\mathrm{S}_{\mathrm{S}}$ değeri sırasıyla 0.25 ve 1.5 olarak alınmıştır. $\mathrm{S}_{\mathrm{s}}$ ' in 0.25 ve 1.5 olması durumuna göre TBDY' de farklı yerel zemin sınıfları için verilen $F_{s}$ değerleri kullanılarak elde edilen $\mathrm{S}_{\mathrm{DS}}$ değerleri de Tablo 3 ' te verilmiştir. Depremli durum için KDD tasarımında deprem yükünün zemin ile yapıya aktarılması ve duvara etki ettirilmesinde kısa periyot tasarım spektral ivme katsayısına $\left(\mathrm{S}_{\mathrm{DS}}\right)$ göre belirlenen statik-eşdeğer yatay deprem katsayısı $\left(\mathrm{k}_{\mathrm{h}}\right)$ ve statik-eşdeğer düşey deprem katsayısı $\left(\mathrm{k}_{\mathrm{v}}\right)$ kullanılmaktadır. $\mathrm{S}_{\mathrm{S}}=0.25,1.50$ değerleri için optimizasyon analizlerinde $6 \mathrm{~m}$ lik gövde yüksekliğine sahip KDD

Tablo 3. Optimizasyon analizlerinde dikkate alınan farklı farklı tasarım durumları

\begin{tabular}{|c|c|c|c|c|c|c|}
\hline \multirow{2}{*}{ Yerel Zemin Sinıfi } & \multirow{2}{*}{$\boldsymbol{\emptyset}_{\mathrm{d}}\left({ }^{\circ}\right)$} & \multirow{2}{*}{$\gamma_{\mathrm{d}}(\mathrm{kN} / \mathrm{m} 3)$} & \multicolumn{2}{|c|}{$\mathbf{F}_{\mathrm{s}}$} & \multicolumn{2}{|c|}{$\mathbf{S}_{\mathrm{DS}}\left(\mathrm{S}_{\mathrm{S} x F_{S}}\right)$} \\
\hline & & & $\mathrm{S}_{\mathrm{s}} \leq 0.25$ & $\mathrm{~S}_{\mathrm{s}} \geq 1.50$ & $\mathrm{~S}_{\mathrm{s}} \leq \mathbf{0 . 2 5}$ & $S_{s} \geq 1.50$ \\
\hline$Z A$ & 38 & 20 & 0.80 & 0.80 & 0.200 & 1.20 \\
\hline$Z B$ & 36 & 19 & 0.90 & 0.90 & 0.225 & 1.35 \\
\hline$Z C$ & 34 & 18 & 1.30 & 1.20 & 0.325 & 1.80 \\
\hline$Z D$ & 30 & 17 & 1.60 & 1.00 & 0.400 & 1.50 \\
\hline$Z E$ & 28 & 16 & 2.40 & 0.80 & 0.600 & 1.20 \\
\hline
\end{tabular}

Optimizasyon analizleri sonucunda 10 farklı tasarım durumu için minimum amaç fonksiyonu değerini veren ve verilen sınırlayıcıları sağlayan optimum KDD boyutları elde edilmiştir. Farklı tasarım durumları için optimizasyon analizleri sonucu elde tasarımlarının depremli ve depremsiz durumdaki taşıma gücü tahkiklerinde aynı temel zemini dikkate alınmıştır.

Tablo 1' de verilen tasarım değişkenleri kullanılarak oluşturulan tasarım kümesi dikkate alınarak başlangıç ve yeni besin kaynakları oluşturulmuştur. Oluşturulan besin kaynakları (çözüm) için Tablo 2' de verilen sınırlayıcılar ve amaç fonksiyonunun değerleri belirlenmiştir.

MR, NEB ve NOB, NFS, limit ve maxiter algoritma parametrelerinin sirasiyla $0.40,30,15, \mathrm{NFSxN}$ ve 1000 olarak alındığı optimizasyon analizlerinde tüm tasarım durumları için algoritma 30 defa çalıştırılmıştır.

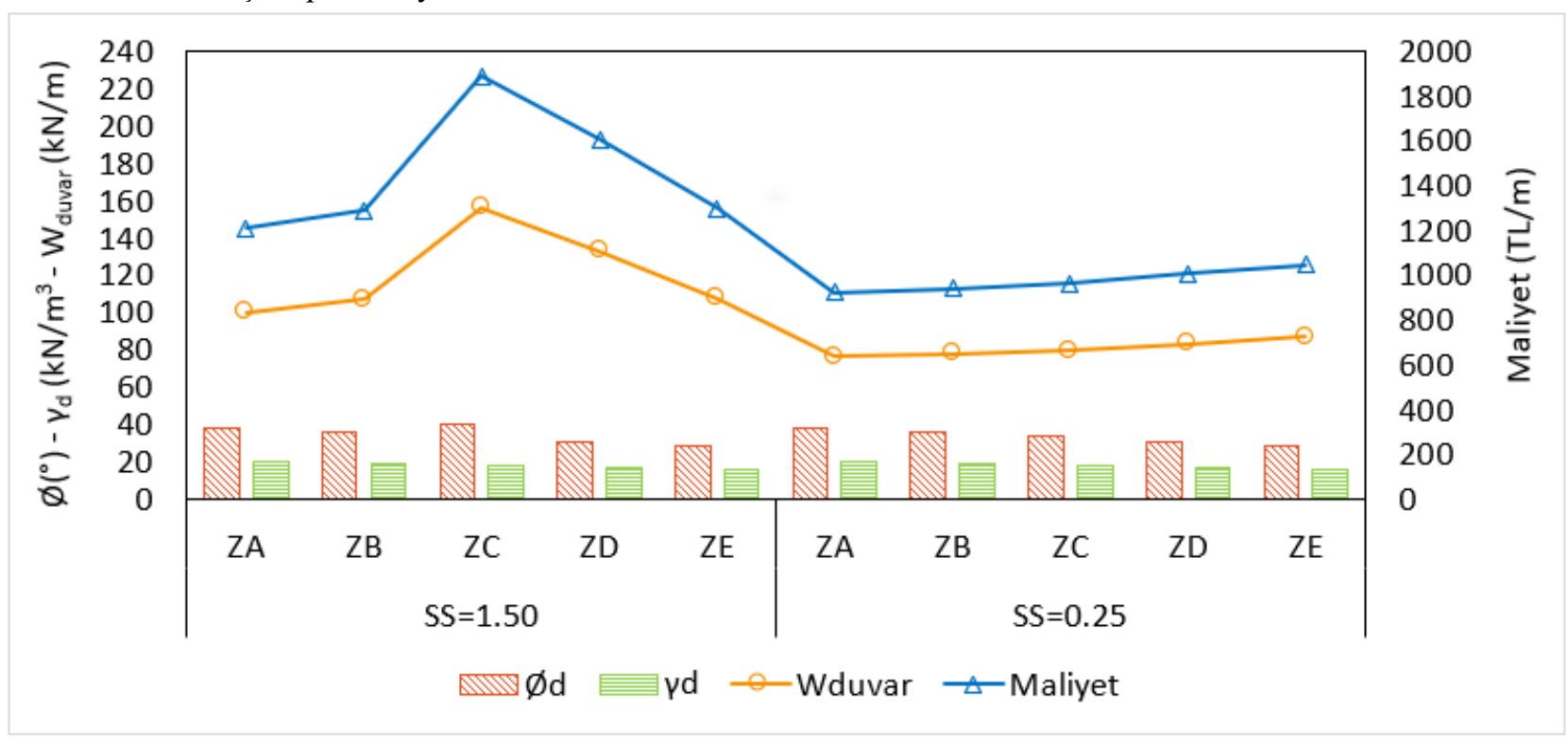

Şekil 2. Farklı tasarım durumları için optimum analiz sonuçları

Şekil 3' te verilen grafik farklı deprem bölgeleri açısından incelendiğinde deprem etkisi fazla olan durumda $\left(\mathrm{S}_{\mathrm{s}}=1.50\right)$ duvar maliyetlerinin deprem etkisi az olan duruma $\left(\mathrm{S}_{\mathrm{S}}=0.25\right)$ göre arttığ görülmektedir. $\mathrm{Bu}$ durum depremli durum için yapılan tasarımlarda stabilite koşullarının sağlanması için boyutların artması sebebiyle maliyetin arttığını göstermektedir. Farklı yerel zemin sınıfları açısından sonuçlar değerlendirildiğinde $\mathrm{Ss}=0.25$ için iyi nitelikli zeminden zayıf nitelikli zemine doğru (ZA' dan ZE' ye doğru) maliyetin arttığı gözlemlenirken, $\mathrm{Ss}=1.50$ için $\mathrm{ZA}$ ' dan $\mathrm{ZC}$ ' ye doğru benzer bir trend görülmektedir. $\mathrm{Ss}=1.50$ ve $\mathrm{ZC}$ yerel zemin sınıfının dikkate alındığ tasarım durumunda $\varnothing_{\mathrm{d}}=34^{\circ}$ edilen KDD optimum sonuçları duvar ağırlık ve maliyet karşılaştırmaları için Şekil 3' te, duvar boyutları karşılaştırmaları için Şekil 4'te verilmiştir. 
ampatman $\left(\mathrm{X}_{4}\right)$ boyutu $\mathrm{S}_{\mathrm{s}}{ }^{\prime}$ in 0.25 ve 1.25 olduğu durumların her ikisinde de stabilite kriterlerinin sağlanması açısından etkili bir parametre olduğu sonucuna varılmıştır. Alt $\left(\mathrm{X}_{3}\right)$ ve üst $\left(\mathrm{X}_{1}\right)$ gövde kalınlıklarının farklı tasarım durumlarında önemli değişim elde edilmezken, temel kalınlığ ${ }_{1}\left(\mathrm{X}_{5}\right)$ için tüm tasarım durumlarında aynı değer elde edilmiştir.

\section{Sonuç}

KDD geleneksel yöntemle tasarımında stabilite kriterlerini sağlayan duvar boyutlarının birçok tasarım durumunu dikkate alarak elde edilmesi uğraştırıcı ve zaman alıcı bir yöntemdir. $\mathrm{Bu}$ çalışmada sezgisel optimizasyon yöntemlerinden biri olan MYAKA ile farklı tasarım durumları için deprem yüklerine maruz KDD’ nın minimum maliyetli ve güvenli tasarımları kısa zamanda ve etkili bir şekilde elde edilmiştir. Ayrıca değişen farklı tasarım parametrelerinin maliyet gibi bir hedef değer üzerinde değişim etkisinin kısa zamanda ve mantıklı olarak yorumlanması imkânı vermesi çok bilinmeyenli tasarım problemlerinin optimum çözümlerinin elde edilmesinde MYAKA sezgisel optimizasyon yönteminin alternatif bir yol olduğu sonucu görülmüştür. Gelecek çalışmalarda, artan bilinmeyen sayısı ile birlikte farklı tasarım kombinasyonlarını kapsayan arama mozaiği kullanılarak çalışmanın kapsamı parametrik bir araştırma ile genişletilebilir.

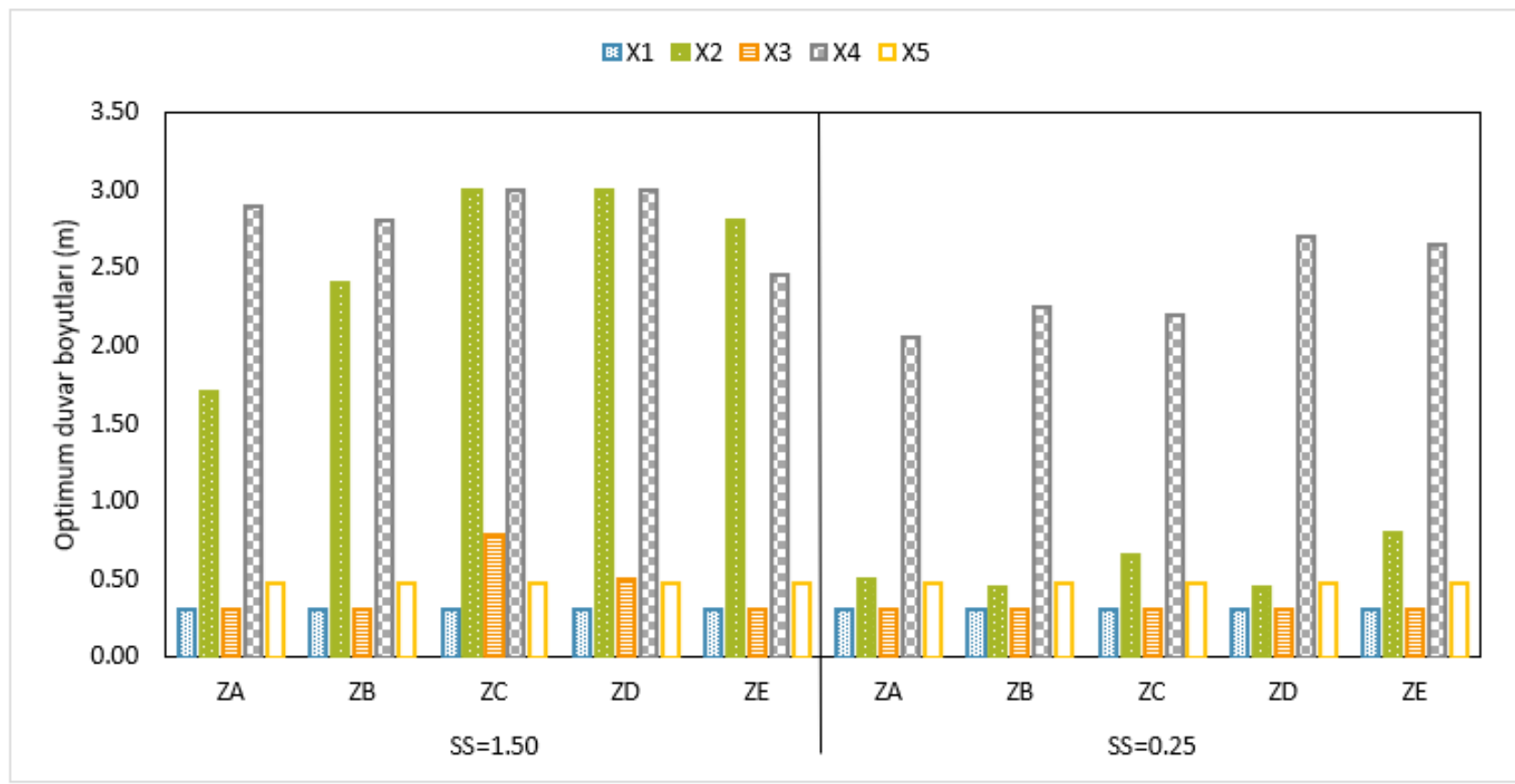

Şekil 3. Farklı tasarım durumları için optimum duvar boyutları

\section{Kaynakça}

Akay, B., \& Karaboga, D. (2012). A modified Artificial Bee Colony algorithm for real-parameter optimization. Information Sciences, 192, 120-142. https://doi.org/10.1016/j.ins.2010.07.015

Arslan, Ö., Keskin, I., \& Ateş, A. (2018). Farklı deprem yüklerinin betonarme konsol bir istinat duvarının maliyetine etkisinin analizi. Anadolu Üniversitesi Bilim Ve Teknoloji Dergisi - B Teorik Bilimler, 6, 28-35. https://doi.org/10.20290/aubtdb.497527

Aydogdu, I. (2017). Cost optimization of reinforced concrete cantilever retaining walls under seismic loading using a biogeography-based optimization algorithm with Levy flights. Taylor \& Francis, 49(3), 381-400. https://doi.org/10.1080/0305215X.2016.1191837

Bilgin, H. (2006). Istinat duvarlarının dinamik ve statik yükler altındaki davranışının analizi [Karadeniz Teknik Üniversitesi].

http://acikerisim.ktu.edu.tr/jspui/bitstream/123456789/1095/ 1/Tam Metin.pdf

Camp, C., \& Akin, A. (2012). Design of retaining walls using big bang-big crunch optimization. Journal of Structural Engineering, 138(March), 438-448. http://ascelibrary.org/doi/abs/10.1061/(ASCE)ST.1943-
$541 \mathrm{X} .0000461$

Ceranic, B., Fryer, C., \& Baines, R. W. (2001). An application of simulated annealing to the optimum design of reinforced concrete retaining structures. Computers and Structures, 79(17), 1569-1581. https://doi.org/10.1016/S00457949(01)00037-2

Chau, K. W., \& Albermani, F. (2003). Knowledge-Based System on Optimum Design of Liquid Retaining Structures with Genetic Algorithms. Journal of Structural Engineering, 129(10),

1312. http://link.aip.org/link/JSENDH/v129/i10/p1312/s1\&Agg=d oi

Dağdeviren, U., \& Kaymak, B. (2018). Investigation of parameters affecting optimum cost design of reinforced concrete retaining walls using artificial bee colony algorithm. Journal of the Faculty of Engineering and Architecture of Gazi University, 33(1), 239-253. https://doi.org/10.17341/gazimmfd.406796

Dalyan, İ., Akın, M., \& Akbay Arama, Z. (2020). Betonarme istinat duvarlarının geoteknik tasarımında etkili parametrelerin 2018 Türkiye Bina Deprem Yönetmeliği’ne göre değerlendirilmesi. Türk Deprem Araştırma Dergisi, 2(2), 176-192. https://doi.org/10.46464/tdad.80428

Das, B. (2007). Principles of Foundation Engineering 6th Edition.

http://thuvienso.hau.edu.vn:8888/dspace/handle/hau/5215 
Deb, K. (2000). An efficient constraint handling method for genetic algorithms. Computer Methods in Applied Mechanics and Engineering, 186(2-4). https://doi.org/https://doi.org/10.1016/S00457825(99)00389-88

Kalemci, E. N., İkizler, S. B., Dede, T., \& Angın, Z. (2020). Design of reinforced concrete cantilever retaining wall using Grey wolf optimization algorithm. Structures, 23, 245-253. https://doi.org/10.1016/j.istruc.2019.09.013

Karaboga, D. (2005). An idea based on honey bee swarm for numerical optimization. Technical Report TR06, Erciyes University, Turkey. https://pdfs.semanticscholar.org/015d/f4d97ed1f541752842c 49d12e429a785460b.pdf

Karaboğa, D. (2014). Yapay zeka optimizasyon algoritmalart. Nobel Akademik Yayıncılık ISBN978-605-133-764-7.

Karaboga, D., \& Akay, B. (2011). A modified Artificial Bee Colony (ABC) algorithm for constrained optimization problems. Applied Soft Computing Journal, 11(3), 30213031. https://doi.org/10.1016/j.asoc.2010.12.001

Kaveh, A., Akbari, H., \& Hosseini, S. M. (2021). Plasma generation optimization for optimal design of reinforced concrete cantilever retaining wall structures. Iranian Journal of Science and Technology - Transactions of Civil Engineering. https://doi.org/10.1007/s40996-020-00566-8

Kayhan, A., \& Demir, A. (2018). Statik ve dinamik yüklere maruz betonarme konsol istinat duvarlarının diferansiyel gelişim algoritması ile optimum tasarımı. Pamukkale Üniversitesi Mühendislik Bilimleri Dergisi, 24(3), 403-412. https://doi.org/10.5505/pajes.2017.04834

Khajehzadeh, M., Taha, M. R., El-Shafie, A., \& Eslami, M. (2010). Economic design of retaining wall using particle swarm optimization with passive congregation. Australian Journal of Basic and Applied Sciences, 4(11), 5500-5507.

Meyerhof, G. G. (1963). Some Recent Research on the Bearing Capacity of Foundations. Canadian Geotechnical Journal, 1(1), 16-26. https://doi.org/10.1139/t63-003

Ravichandran, N., Wang, L., Rahbari, P., \& Juang, C. H. (2021). Robust design optimization of retaining wall backfilled with shredded tire in the face of earthquake hazards. Bulletin of Engineering Geology and the Environment, 80(2), 13511363. https://doi.org/10.1007/s10064-020-02038-9

Sheikholeslami, R., Khalili, B., Of, S. Z.-I. J., \& 2014, U. (2014). Optimum cost design of reinforced concrete retaining walls using hybrid firefly algorithm. Ijetch.Org. https://doi.org/10.7763/IJET.2014.V6.742

TDBY. (2018). Türkiye bina deprem yönetmeliği. Afet ve Acil Durum Yönetimi Başkanlı̆̆ 1 , Ankara. http://www.resmigazete.gov.tr/eskiler/2018/03/20180318M1 .pdf

TDTH. (2018). Türkiye Deprem Tehlike Haritalart. İnteraktif Web Uygulaması. https://tdth.afad.gov.tr/TDTH/main.xhtml

Temur, R., \& Bekdas, G. (2016). Teaching learning-based optimization for design of cantilever retaining walls design and analysis of nonlinear structural systems view project teaching learning-based optimization for design of cantilever retaining walls. Structural Engineering and Mechanics, 57(4), 763-783. https://doi.org/10.12989/sem.2016.57.4.763

Uray, E., Tan, Ö., Çarbaş, S., \& Erkan, H. (2021). Metaheuristicsbased pre-design guide for cantilever retaining walls. Teknik Dergi, 32(4). https://doi.org/10.18400/tekderg.561956

Yıldırım, İ. Z. (2004). İstinat Duvarlarının Tasarımında Deprem Etkilerinin İncelenmesi [İstanbul Teknik Üniversitesi].
https://tez.yok.gov.tr/UlusalTezMerkezi/tezSorguSonucYeni. jsp 